\title{
Increasing Students' Interest With Low-Cost CellBots
}

\author{
Rafael V. Aroca, Member, IEEE, Rafael B. Gomes, Dalton M. Tavares, Anderson Abner S. Souza, \\ Aquiles M. F. Burlamaqui, Glauco A. P. Caurin, Member, IEEE, and Luiz M. G. Gonçalves, Member, IEEE
}

\begin{abstract}
This paper introduces the use of a flexible and affordable educational robot specifically developed for the practical experimentation inherent to technological disciplines. The robot has been designed to be reconfigurable and extendible, serving as an experimental platform across several undergraduate courses. As most students have a mobile cell phone, this was used as the main control computer for the so-called CellBot, thus avoiding any need to deal with the details of microcontrollers or other embedded computing devices. Assessment results are also presented, based on a pre- and post-survey of student opinion administered to 204 science and engineering students from several universities. Among the conclusions are that $83 \%$ of the students prefer to use these low-cost robots as tools to improve their learning of the theory in several disciplines, and $71 \%$ of the students stated that they prefer to have their own robot to experiment with, instead of using a didactic kit loaned to them by the university.
\end{abstract}

Index Terms-Audio interface, CellBots, engineering education, low-cost robotics, robotics education.

\section{INTRODUCTION}

$\mathbf{R}$ OBOTICS is a fascinating discipline that easily engages engineering students. As robots in education are stimulating and motivating [1]-[4], there are good reasons for introducing robotics activities very early in course curricula, allowing students to easily perceive the relationships between undergraduate courses, in their theory and practice. Robotics also offers a good basis for teaching different engineering disciplines [5]. Moreover, Weinberg and Xudong argue that "robotics provides a unique learning experience" [6].

The authors of this paper have noticed that when students have the opportunity of experimenting with robots, they often express a desire to have their own robots on which to experiment further at home. While they are ready to invest their free time and money to build robots, few undergraduate students can spend much money on such projects. To address this constraint, this paper proposes a low-cost robot that uses the students' mobile phones as control computers, allowing them to build their own robots. As the phone is used as the robot's computer, only

Manuscript received May 21, 2012; accepted August 07, 2012. Date of publication September 07, 2012; date of current version January 30, 2013. This work was supported by the National Research Council (CNPq), Brazilian Sponsoring Agency for Research.

R. V. Aroca, R. B. Gomes, A. M. F. Burlamaqui, and L. M. G. Gonçalves are with the Federal University of Rio Grande do Norte (UFRN), Natal 59078-900, Brazil (e-mail: rafaelaroca@ieee.org; rafaelufrn@gmail.com; aquiles@natalnet.br; 1marcos@computer.org).

D. M. Tavares is with the Federal University of Goias (UFG), Catalão 75704020, Brazil (e-mail: dalton.tavares@catalao.ufg.br).

A. A. S. Souza is with the State University of Rio Grande do Norte (UERN), Mossoró 59088-100, Brazil (e-mail: andersonabner@uern.br).

G. A. P. Caurin is with the University of São Paulo (USP), São Carlos 13566590, Brazil (e-mail: gcaurin@sc.usp.br).

Digital Object Identifier 10.1109/TE.2012.2214782 a simple analog circuit is needed, and there is no need to use microcontrollers.

This scheme allows the robot to take advantage of all the sensors and features of modern cell phones, which are frequently equipped with a Global Positioning System (GPS) receiver, camera, 3-axis compass and accelerometer, Wi-Fi, Bluetooth, speakers, microphone, and several other features that fit perfectly in a robot control system. Also, it has been already shown that it is feasible to execute complex robotics navigation algorithms using the processors of mobile phones [7]. These interesting possibilities were confirmed in a recent report in IEEE Robotics and Automation Magazine identifying "Smartphone-based robots: The new robot brains" as one of the top robotics trends for 2012 [8].

During this research, 204 science and engineering students were tracked in their several undergraduate courses during one semester to evaluate their interest in using low-cost robots. More than $70 \%$ said that they would spend their money to build their own robot if it could be used in several courses. The main contribution of this paper is the application of a flexible and low-cost robot adapted from a previous work [9], disseminating the use of robotics for educational purposes. Also provided are a survey of state-of-the-art low-cost educational robots and an assessment of Brazilian students' interest in robotics-related subjects in this low-cost context.

The rest of this paper is organized as follows. Section II reviews the state of the art in related fields, presenting several works dealing with robotics in education. Section IV presents the material and methods used in this research. Section III presents some possible setups for low-cost robotics in educational environments. Next, Section V presents and discusses the results of applying the proposed platform in regular courses at several Brazilian universities. Finally, Section VI discusses the results, draws conclusions, and points future directions.

\section{STATE OF THE ART}

Robotics is an ideal solution for curriculum integration, allowing students to use and integrate concepts learned in several disciplines [10], [11]. In some cases, courses are created specifically to use robots to teach several topics [1]. Teamwork and multidisciplinary team skills are also enforced [11]. Furthermore, most people learn more easily when hands-on practice is involved in learning activities [12], therefore robotics can be used as a pedagogical tool that offers a "learning by doing" experience [3], [13]. In what follows, several low-cost robots built for these educational purposes are described.

\section{A. Low-Cost Educational Robots}

Although robots are a great educational tool, Alves et al. warn that the high cost of robots can prevent their being used in classrooms [3], [14]. In the same way, Lumsden and Ortega-Sanchez say that there is a lack of inexpensive and flexible mobile robotic 
platforms [15]. This reality led educators to propose several low-cost educational robots [2], [14]-[17] for teaching.

There are several articles in the literature describing low-cost robot designs for education; some of them are directly related to this work. Many propose low-cost educational robots, but in most cases without actually giving these costs. Other authors propose low-cost educational robots costing between $\$ 200$ and $\$ 1000$ USD [2], [15]-[17]. That might be a low cost for universities, but is expensive for most students who want to own a robot or a robotics kit, especially those in developing countries.

Piperidis et al. report a survey showing that only 5\% of commercially available mobile robots cost less than 100 Euros (about \$133 USD in April 2012). They further state that a robot suitable for both educational and research needs costs at least 3000 Euros (about \$3972 USD). In conclusion, they say that there is a need for mobile robots with better cost benefit and propose a 500 Euro (about \$662 USD) mobile robot [18]. As an alternative, Hamblen and Hall say that it is possible to keep the costs of a robot affordable for students if toy parts are used [2].

A common approach used in several cases is the use of LEGO kits [11], which are easy to use, but have limited flexibility and cost several hundred dollars. Another common solution found in robots made by students is the use of laptops as robot controllers, but unfortunately, laptop computers have limited I/O interfaces, so auxiliary circuit boards must be used to connect sensors [2] and actuators, and the robot must be robust if it is to carry a laptop.

One solution that is gradually gaining more attention is the use of smartphones as the main controllers of low-cost robots [7]-[9], [19]. As mobile phones have several features and sensors directly useful for robots, operating them as a robot's main processing unit is an interesting option. Moreover, as they are produced on a very large scale, it is cheaper to buy a mobile phone with built-in accelerometer, compass, camera, and other sensors than to buy a microcontroller and all these sensors separately and then integrating them. Finally, smartphones provide simple development environments and application programming interfaces (APIs) that are easier to use than those provided by microcontroller manufacturers.

\section{B. Robots as Motivators}

It is a common belief that better learning happens when students are engaged and motivated [14], [20], [21]. According to Alves et al., robotics can be used as motivating element [3], and Rawat and Massiha verify that student feedback after robotic classes is "overwhelmingly positive" [5]. Alves et al. also relate that students find building robot prototypes a motivating activity [3]. Moreover, students show a deep interest in the application of concepts learned during classes featuring robots [14].

Assessment of robotics-based practical work in various disciplines show that students consider these laboratory practices challenging and stimulating [22]. Firebaugh and Piepmeier collected student feedback about a course on nanorobots; most rated the course as "one of the best" they had taken. Their students also say that "working in a laboratory" and "making something" are strong features of that specific robotics course [23].

One study shows that students are more motivated by the desire for learning than by gaining a prize or credits for completing a course [24]. This result is consistent with the theories described in Pink's book [21] that compiles several studies on motivation. According to Pink, autonomous motivation, which comes from individual interest "promotes greater conceptual understanding, better grades, enhanced persistence at school..." [21]. He also argues that this kind of motivation leads to engagement.

One important issue in motivation is that the tasks must be neither too difficult nor too easy, otherwise the motivation is lost [20], or even worse, the tasks can cause frustration [21]. According to Mok, a mismatch of task complexity and students' skills hampers them from becoming deeply engaged [20]. CellBots are interesting solutions to this problem because they allow a wide variety of topics and difficulties to be explored in a single platform, suitable for freshmen and senior students.

\section{Low-COST EDUCATIONAL RoBOT}

The Lumsden/Ortega-Sanchez robot [15] offers good cost/ benefit. For about $\$ 755$ USD, the system contains a $20-\mathrm{MHz}$ microcontroller, $4 \mathrm{kB}$ of RAM memory, GPS, a 2-axis compass, servos, electronics boards, liquid crystal display (LCD), and motors. The differential drive robot used in this work can be built for less than $\$ 15$ using two model airplane micro servomotors to drive the wheels. A smartphone is used as the control computer, featuring a 1-GHz dual-core processor, $1 \mathrm{~GB}$ of RAM memory, gyroscope, 3-axis accelerometer and compass, high-resolution camera, Internet connectivity using 3G/GPRS data services or $\mathrm{Wi}-\mathrm{Fi}$, and other features.

If the student already has this kind of phone, she/he will only have to spend a few dollars, but even if a mobile phone has to be purchased, the entire robot, including the mobile phone, would cost less than half of the $\$ 755$ USD robot, but with same or superior processing, communication, and sensing features. Also, it would run the Android or Linux operating system, giving the students a flexible environment to experiment with robotics, distributed systems, operating systems, Java programming, computer vision, and other topics met in related courses.

A common approach to building a CellBot is to use a microcontroller connected to the mobile phone, but here, instead of using microcontrollers and digital interfaces such as Bluetooth, USB, or RS-232 ports, the phone controls the actuators directly, using a standard and universal communication channel: the phone's audio output. The control circuit needed is quite simple to build, even for freshmen. It is also possible to implement closed-loop control using digital signal processing (DSP) techniques: Each sensor generates a different audio tone and sends it to the mobile phone audio input (microphone), which decodes these tones using software to identify sensor states. As this system uses only simple analog hardware, it is possible to build a differential drive mobile robot for less than \$15 USD. Wheel encoders and closed-loop control can be implemented for less than \$30 USD.

Although the focus here is on the use of mobile phones, any device that can produce sounds could be used as the robot controller. Therefore, a tablet computer, netbooks, or even standard computers can control and read sensors by using their audio input and output.

As the focus of this paper is educational, a summary of the technical aspects of the system follows here, but without further details, which can be found in the references provided. 


\section{A. Open Loop}

To build a robot with open-loop control, two hobby servos with continuous rotation are used, one for each wheel. The servos are connected to a battery, and the audio output of the mobile phone is directly connected to an optical coupler, which has its output connected to the pulse width modulation (PWM) input of the servos. The left audio channel is connected to the left servo, and the right audio channel is connected to the right servo. By generating a sound that mimics a PWM signal, software running on the mobile device can control each wheel independently to go forward, backward, or at any intermediate speed between the maximum forward and backward speeds. Cheap satellite dish servos can also be used in this same setup. For a detailed explanation of this setup, which has already been used in several projects, see [19] or check the NatalNet laboratory Web page [25].

If more powerful motors are needed, the audio outputs can be used to control an H-bridge motor driver, which allows the control of several kinds of dc motors. A reliable solution is to use dial tone multifrequency (DTMF) audio signals to easily control up to four dc motors using stereo audio interfaces of mobile devices [9].

Although this section only describes how to control motors, a control loop can be closed using the mobile phone's built-in sensors. For example, it is possible to use the mobile device's compass or gyroscope to control the robot heading direction and curves [9]. Moreover, the accelerometer and computer vision can be used to compute odometry by using visual odometry techniques and to implement simultaneous localization and mapping (SLAM) techniques. Computer vision can also be used to replace ultrasound and infrared distance sensors with good precision [9].

Among courses that can take advantage of this technique, and thus motivate students, are those in computer networks (to implement remote control), operating systems (to study Linux and Android in detail for robot control purposes), real-time systems, distributed systems, object-oriented programming, computer vision, and others.

\section{B. Closed Loop}

The system proposed here can also be used if external sensors such as bumpers and wheel encoders are needed. A DTMF encoder generates various DTMF signals in response to the various states of each sensor. The mobile phone then samples the incoming audio and uses a Fourier transform to identify which tones are present in the audio signal. After decoding these tones, the information is interpreted as sensor states. A detailed explanation of this method can be found in [9], which also shows that this system allows control loops to be closed with $50-\mathrm{Hz}$ update rates, similar to the sampling performance of LEGO Mindstorms' NXT sensors.

Implementing these techniques gives students a practical and motivating opportunity to learn important concepts of digital signal processing, such as Fourier transforms, sampling rate theorems, and signal synthesis and analysis. It is also a "playground" for control theory that the student can take home to experiment with proportional $(\mathrm{P})$, proportional-integral $(\mathrm{PI})$, and proportional-integral-derivative (PID) controllers in many different forms.
As the robot can be used in a variety of courses, the teachers can provide software that best fits each discipline to help students focus on a specific topic to be learned. For example, in a DSP course, fast Fourier transform (FFT) implementations might be omitted to allow students to write their own versions, while these might be provided in other courses.

A common and powerful approach used with commercial educational equipment is to use MATLAB/Simulink to implement control models and other systems graphically, and then automatically generate code, thus avoiding the need for students to write code for certain tasks. This approach has been demonstrated with the proposed system, which allows a user to design systems using Simulink blocks and then automatically generate code that will run on a mobile device.

\section{MATERIAL AND Methods}

First, an anonymous survey was given to all students to elicit their conceptions of and interests in robotics, as well as information about their mobile phones. Some weeks later, they were given a lecture, "How to build your own low-cost robot using the mobile phone as its brain." Student verbal or e-mail feedback on the lecture was documented, and a post-survey was administered to assess changes in their conceptions or levels of interest and motivation.

The survey was composed of multiple-choice questions on their interest in undergraduate courses, robotics concepts, their mobile phone's characteristics, and their interest in building or buying a robot.

It was completed by 204 students in four Brazilian universities, aged from 18 to 53 years old (average 21). These students are enrolled in Mechatronics Engineering, Computer Engineering, Electrical Engineering, Computer Science, and Science and Technology undergraduate courses. After the robotics lecture, a Ph.D. student was available for several weeks to help any student interested in building his/her own robot.

\section{RESUlts AND DisCUSSION}

\section{A. Preliminary Survey}

The preliminary survey results show that $41.5 \%$ of all students said that they prefer to use a university kit instead of buying or building their own robots. The remaining $58.5 \%$ said that they would prefer to have their own robots (of these students, $78 \%$ would prefer to build their own robots, while $22 \%$ would prefer to build a ready-to-use kit). Moreover, 23\% of these students said that they would not spend any money on an educational robot or on a robotic kit, $13 \%$ would spend $\$ 25$ or less, $27 \%$ would spend up to $\$ 50,18 \%$ would spend up to $\$ 125$, and $19 \%$ would spend $\$ 250$ or more.

Only $1 \%$ of the students did not have a mobile phone. $27 \%$ had purchased their phones in the last six months, $32 \%$ in the last year, $21 \%$ in the last two years, and $19 \%$ in the last four years. Regarding the phone features, $68 \%$ have cameras, $32 \%$ have GPS receivers, $27 \%$ have accelerometers, $47 \%$ have wireless Wi-Fi connections, $68 \%$ have Bluetooth modules, and $94 \%$ have earphone outputs.

They were also asked which courses are most important for learning robotics; their responses by course were the following: DSP, 67\%; Control, 62\%; Introduction to Robotics, 83\%; Linear Algebra, 42\%; Computer Vision, 55\%; Neural Networks, 34\%; 


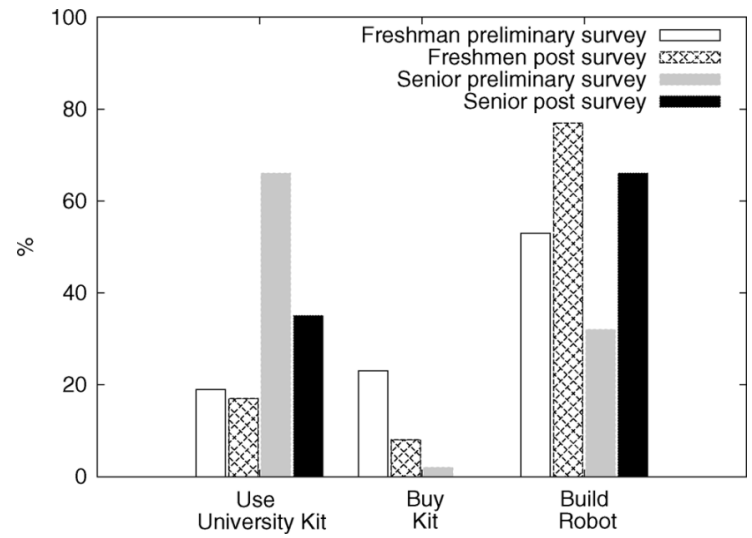

Fig. 1. Students interests in building a robot or using an off-the-shelf kit for freshmen (classes of 2010 and 2011) and senior students (class of 2007).

Statistics, 19\%; Stochastic Processes, 14\%; Calculus, 45\%; Object-Oriented Programming, 60\%; Web Programming, 25\%; Computer Networks, 35\%; Distributed Systems, 34\%; Operating Systems, 48\%; and Real-Time Systems, 53\%.

\section{B. Post-Survey}

The post-survey was applied to assess if students' opinions changed after learning they could build their own low-cost CellBot. Of the sample of 204 students, $83 \%$ said they thought it would be more interesting to learn several topics using a CellBot to apply the theory they had learned (for the population, the average estimate is in the interval from $76 \%$ to $89 \%$ with a confidence level of $95 \%$ ). While in the pre-survey, $58.5 \%$ of the students said that they would prefer to own their own robots, this number increased to $71 \%$ in the post-survey, showing that using a CellBot increased the overall student interest (for the population, the average estimate is in the interval from $64 \%$ to $77 \%$ with a confidence level of $95 \%$ ).

Fig. 1 shows the level of student interest in building their own robots, before and after the lecture. The results are divided into two categories: freshmen students who entered the university in 2010 or 2011, and seniors who entered the university in 2007. From the figure, it is clear that freshmen were divided, probably because they did not know what options were available, while senior students were more strongly inclined to use kits already available at the university. Fig. 1 also shows that after learning of the CellBot possibility, many students from both groups migrated to the option of building their own robot. One interesting change was observed in a Computer Science class, where the interest of the students in building robots rose from $0 \%$ to $59 \%$.

As shown in Fig. 2, after the robotics lecture, the percentage of students inclined to spend money to build their own robots increased. Before the robotics lecture, the students were less interested in investing money in a robot, but after the lecture, more students said they were willing to spend US $\$ 25$ or US $\$ 50$ or even more on a robot. It is interesting to note that the peak of the plot is at US $\$ 50$, near the proposed value of the CellBot.

The surveys also asked students about their interest in undergraduate courses, but except for the DSP course, no significant difference was seen in their interest in pursuing specific disciplines. The freshmen's interest in pursuing DSP increased from $83 \%$ to $95 \%$ after the lecture. Clearly this

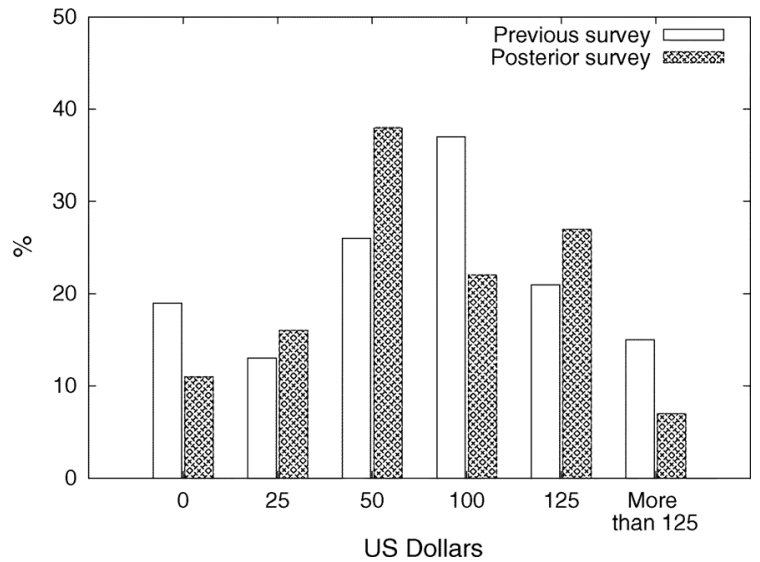

Fig. 2. Distribution of how much the students are willing to spend to build their robots (values in US dollars).

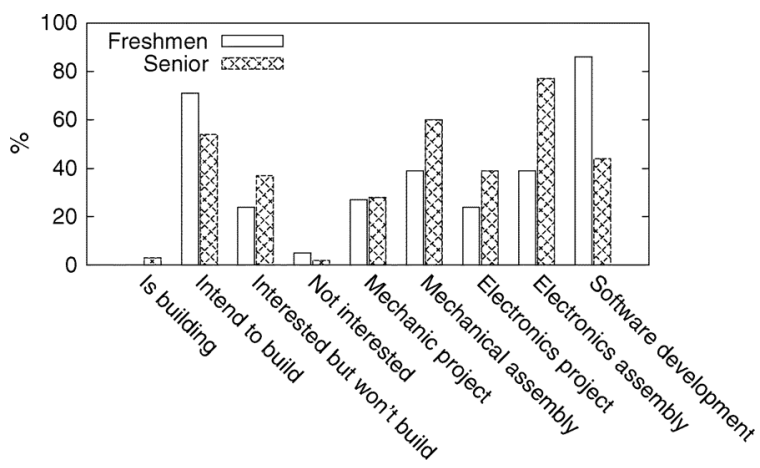

Fig. 3. Students' interest in CellBots after the post-survey.

happened because the robotics lecture showed the importance of DSP techniques in building the proposed CellBot (due to the audio signal processing requirements). Interestingly, for the senior students, $57 \%$ had already taken the DSP course, but $8 \%$ were not interested in such a course. Many of these senior students had already taken DSP classes that took a strong theoretical approach and where they met few implemented real DSP systems during the classes. As the lecture showed a clear and practical application of DSP concepts, freshmen students promptly became more interested in this discipline.

Fig. 3 shows the relative levels of the students various interests in the low-cost CellBot. As the post-survey was applied several weeks after the low-cost robot lecture, some of the students were already building their own robots (about $8 \%$ ). As shown in Fig. 3, the majority of students became interested in the project or intend to build their own robots. Apparently, senior students were also interested in the project, but less interested in building the robot, mainly because they were at the end of their course and already looking for other challenges after graduation. Moreover, there are fewer concepts to learn and challenges for these students. Fig. 3 also shows the various levels of interest in the mechanical project, assembly, electronics, and software development aspects of the robot.

It is interesting to note the considerable change in student opinion after just one 40-min class describing the low-cost CellBot option. Their level of motivation and interest would be even higher if such a "take home" low-cost robot was a widely used tool to learn and test concepts in other courses. The CellBot has wide application, even in courses that are not 
directly related to robotics. Web programming can be used to implement Web-based robot remote control, calculus to show the basis for closed-loop control systems such as PID, linear algebra for building homogeneous transform matrices for robot kinematics computation, and so on.

\section{Qualitative Assessment}

The last survey gave the students an opportunity to make comments, some of which are given here along with some observations by the authors. Some comments and feedback were surprising: After giving the lecture in one of the universities, students asked for a regular robotics course in their Computer Science curriculum (this university did not have any robotics courses), and the committee accepted the challenge and created the new course. Some students wanted to do ambitious projects - for example, one group of students attempted the development of a quadrotor controlled only by a mobile phone, which has yet to succeed. In fact, Mirats and Pfeiffer report that students tend to propose projects that are much too ambitious to be done in one semester [11]

One rewarding result was observed with students who decided to build robots. For example, a group with two students assembled a functional system in about $2 \mathrm{~h}$. Interestingly, they had never used a soldering iron, showing that even inexperienced students can build such a robot. After they saw the robot's motor under the mobile phone control, they got interested in improving the control software with additional features.

Some students asked for courses or mini-courses on the CellBot's construction. One of the students said that the "concept of robotics should be added in undergraduate courses, given that the topic causes great curiosity and interest." Another student said that "this should be released more broadly because it is very interesting and essential for undergraduate students."

Others said that robotics should be studied more in universities, and that the CellBot is a good option because it is easy to build and can be easily used in a course, giving the students the opportunity to learn basic techniques to build robots.

Another student suggested bulding versions of the system that can be controlled with simpler cell phones, even if there are not many features on such phones. This has actually been done by one of the students of another university involved in this research. This student created a Java 2 Micro Edition (J2ME) version of the system that can control the robot and receive commands from the network. Other students are now working on a line-following robot based on the camera of a cheap mobile phone. J2ME is a lightweight version of Java specifically for low-cost devices with constrained hardware resources. Most cheap cell phones on the market currently support J2ME.

Fig. 4 shows the reference robot built by a Ph.D. student and shown to the undergraduate students as a working model so they could see a functional CellBot and its parts to help them in making the design decisions for their own CellBots. Fig. 5 shows a CellBot built by a Computer Engineering student using a solderless prototype board as the frame of the robot and two servomotors controlled by the audio from a mobile phone (not shown in the photograph) to move the robot.

Lecture slides, video, some building instructions, and electrical schematics for the CellBot described in this paper are freely available at the NatalNet laboratory Web page [25].

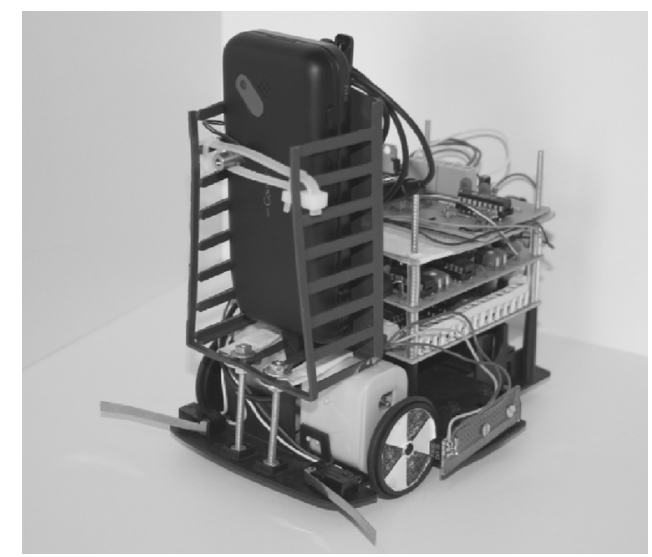

Fig. 4. Reference robot shown to the undergraduate students as a working model for their robots.

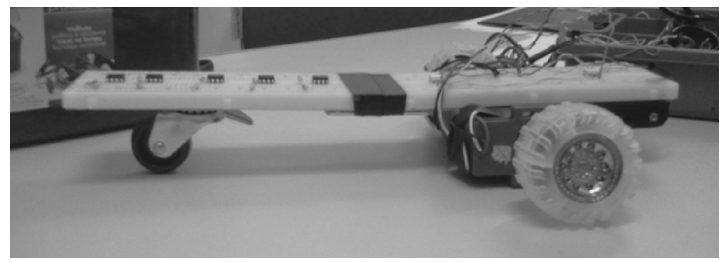

Fig. 5. CellBot built by a Computer Engineering student (phone not shown). Image credit: A. P. Dantas de Araújo.

\section{CONCLUSION}

This paper proposes the use of a very low-cost educational robot system that can be easily built and used by undergraduate students in several courses in their degree program. To keep the cost low, it is assumed that the student has a mobile phone with an earphone output. The students do not need to learn to program microcontrollers or build circuits with microcontrollers. This simplicity allows even freshmen students to start developing and testing systems in this robot. Later, if needed, the architecture allows the system to be connected to microcontrollers and other devices using USB or Bluetooth ports available in the mobile phone or even using the Android Open Accessory Development Kit, which allows microcontrollers to be easily connected to mobile phones.

As the communication between the mobile robot's sensors/ actuators and the control system is based on an audio interface, not only mobile phones can be used, but also any other device that can produce sounds, even an MP3 player or a standard PC, can be used as a control unit. This can be integrated with social-educational projects such as "One laptop per student," promoting the project "one robot per student."

From the assessment, it has been shown that most students are interested in having their own robots and, once they know that it is possible to build a powerful low-cost robot, they are willing to spend their own money to invest in such an educational tool. It has also been shown that at least half of the students do have mobile phones with features and sensors well suited to building a powerful mobile robot. Also, after learning about the CellBots, $71 \%$ of the students became interested in having their own robots, while $83 \%$ of the students said that the use of a CellBot would make the study of other courses easier. As robotics is an engaging and motivating tool for students, the 
method described allows students to have their own robots and use them as a tool for a variety of courses. Future work is aimed at using CellBots throughout the entire undergraduate program.

\section{ACKNOWLEDGMENT}

The authors would like to thank Prof. P. J. Alsina, Prof. L. E. C. Leite, and Prof. D. P. F. Pedrosa for opening their classes for this research. The authors also thank the help of R. Pitta and R. Gardiman for collecting and tabulating data.

\section{REFERENCES}

[1] A. Soto, P. Espinace, and R. Mitnik, "A mobile robotics course for undergraduate students in computer science," in Proc. 3rd IEEE LARS, Santiago, Chile, 2006, pp. 187-192.

[2] J. Hamblen and T. Hall, "Engaging undergraduate students with robotic design projects," in Proc. 2nd IEEE DELTA, Perth, Australia, 2004, pp. 140-145.

[3] S. Alves, H. Filho, R. Pegoraro, M. Caldeira, J. Rosario, and W. Yonezawa, "Proposal of educational environments with mobile robots," in Proc. IEEE RAM, Qingdao, China, 2011, pp. 264-269.

[4] A. Howard and E. Graham, "To encourage and excite the next generation of engineers through human-robot interaction projects for space exploration," presented at the ASEE Annu. Conf., 2007.

[5] K. Rawat and G. Massiha, "A hands-on laboratory based approach to undergraduate robotics education," in Proc. IEEE ICRA, New Orleans, LA, 2004, vol. 2, pp. 1370-1374.

[6] J. Weinberg and X. Yu, "Robotics in education: Low-cost platforms for teaching integrated systems," IEEE Robot. Autom. Mag., vol. 10, no. 2, pp. 4-6, Jun. 2003.

[7] A. C. Santos, L. Tarrataca, and J. M. Cardoso, "The feasibility of navigation algorithms on smartphones using J2ME," Mobile Netw. Appl., vol. 15 , no. 6 , pp. 819-830, Dec. 2010.

[8] E. Guizzo and T. Deyle, "Robotics trends for 2012 [the future is robots]," IEEE Robot. Autom. Mag., vol. 19, no. 1, pp. 119-123, Mar. 2012.

[9] R. V. Aroca, A. F. Burlamaqui, and L. M. G. Gonçalves, "Method for reading sensors and controlling actuators using audio interfaces of mobile devices," Sensors, vol. 12, no. 2, pp. 1572-1593, Feb. 2012.

[10] R. Manseur, "Development of an undergraduate robotics course," in Proc. 27th Annu. Frontiers Educ. Conf., Pittsburgh, PA, 1997, vol. 2, pp. 610-612.

[11] J. Mirats Tur and C. Pfeiffer, "Mobile robot design in education," IEEE Robot. Autom. Mag., vol. 13, no. 1, pp. 69-75, Mar. 2006.

[12] J. Conrad, "Stiquito for robotics and embedded systems education," Computer, vol. 38, no. 6, pp. 77-81, May 2005.

[13] D. Ahlgren, "Meeting educational objectives and outcomes through robotics education," in Proc. 5th Biannu. WAC, Orlando, FL, 2002, pp. 395-404.

[14] S. Galvan, D. Botturi, A. Castellani, and P. Fiorini, "Innovative robotics teaching using LEGO sets," in Proc. IEEE ICRA, Orlando, FL, 2006, pp. 721-726.

[15] J. Lumsden and C. Ortega-Sanchez, "Modular autonomous robotics platform for educational use," in Proc. IEEE TENCON, Fukuoka, Japan, 2010, pp. 1577-1582.

[16] L. Greenwald and J. Kopena, "Mobile robot labs," IEEE Robot. Autom. Mag., vol. 10, no. 2, pp. 25-32, Jun. 2003.

[17] H. Dinh and T. Inanc, "Low cost mobile robotics experiment with camera and sonar sensors," in Proc. ACC, St. Louis, MO, 2009, pp. 3793-3798.

[18] S. Piperidis, L. Doitsidis, C. Anastasopoulos, and N. Tsourveloudis, "A low cost modular robot vehicle design for research and education," in Proc. IEEE MED, Athens, Greece, 2007, pp. 1-6.

[19] R. V. Aroca, A. P. B. S. de Oliveira, and L. M. G. Gonçalves, "Towards smarter robots with smartphones," in Robocontrol: Proc. 5th Workshop Appl. Robot. Autom., Bauru, Brazil, 2012, pp. 1-6.

[20] H. N. Mok, "Student usage patterns and perceptions for differentiated lab exercises in an undergraduate programming course," IEEE Trans. Educ., vol. 55, no. 2, pp. 213-217, May 2012.

[21] D. H. Pink, Drive: The Surprising Truth About What Motivates Us. New York: Riverhead, 2009.
[22] K. Kramer and M. Anderson, "A pic18 robot-centered microcontroller systems laboratory," in Proc. FIE, Rapid City, SD, 2011, pp. T2G-1-T2G-6.

[23] S. Firebaugh and J. Piepmeier, "The Robocup nanogram league: An opportunity for problem-based undergraduate education in microsystems," IEEE Trans. Educ., vol. 51, no. 3, pp. 394-399, Aug. 2008.

[24] M. Hernando, R. Galan, I. Navarro, and D. Rodriguez-Losada, "Ten years of cybertech: The educational benefits of bullfighting robotics," IEEE Trans. Educ., vol. 54, no. 4, pp. 569-575, Nov. 2011.

[25] R. V. Aroca, "CellBots: Personal Webpage of Rafael V. Aroca-Natalnet Laboratory," 2012 [Online]. Available: http://www.natalnet.br/ $\sim$ aroca/

Rafael V. Aroca (M'05) received the Informatics degree and Master of Sciences degree in mechatronics engineering from the University of São Paulo (USP), São Paulo, Brazil, in 2005 and 2008, respectively, and is currently pursuing the Ph.D. in electrical and computing engineering at the Federal University of Rio Grande do Norte (UFRN), Natal, Brazil.

His main research interests are in embedded systems and robotics.

Rafael B. Gomes graduated in computer science in 2007, received the Master's degree in computer engineering from the Federal University of Rio Grande do Norte (UFRN), Natal, Brazil, in 2009, and is currently pursuing the Ph.D. degree with expertise in computer vision, image processing, and robot vision at UFRN.

Dalton M. Tavares received the Bachelor's degree in computer science from the Universidade Estadual Paulista (UNESP), São Paulo, Brazil, in 1999, and the Master's degree in network security and Ph.D. degree in mechatronic systems from the University of São Paulo (USP), São Paulo, Brazil, in 2002 and 2010, respectively.

He currently is performing research on operating systems, networks, computer security, and mechatronics systems.

Anderson Abner S. Souza received the Bachelor's degree in computer engineering and Master's and Doctoral degrees in electrical and computer engineering with focus on robotics from the Federal University of Rio Grande do Norte (UFRN), Natal, Brazil, in 2005, 2008, and 2012, respectively.

Currently, he teaches at The State University of Rio Grande do Norte, Mossoró, Brazil.

Aquiles M. F. Burlamaqui received the Master's and Ph.D. degrees in computer science from the Federal University of Rio Grande do Norte (UFRN) Natal, Brazil, in 2004 and 2008, respectively.

Currently, he is a Professor with UFRN and a Researcher with NatalNet Laboratory, Natal, Brazil, with interests in several fields, including digital TV and educational robotics.

Glauco A. P. Caurin (M'94) graduated in mechanical engineering from the University of São Paulo (USP), São Paulo, Brazil, in 1988, and received the Doctorate degree in subject robotics from ETH Zurich, Zurich, Switzerland, in 1995.

He is an Associate Professor with the Mechanical Engineering Department EESC-USP. During 2011, he was a Visiting Professor with the Massachusetts Institute of Technology (MIT), Cambridge. He works in mechatronic design, robotic manipulation, RTOS, robot rehabilitation systems, and process automation.

Luiz M. G. Gonçalves (M'01) received the Doctorate degree in systems and computing engineering from the Federal University of Rio de Janeiro, Rio de Janeiro, Brazil, in 1999.

$\mathrm{He}$ is an Associate Professor with the Computing Engineering and Automation Department of the Federal University of Rio Grande do Norte (UFRN), Natal, Brazil. His research interests are in computer vision, robotics, and graphics processing.

Dr. Gonçalves is a member of the IEEE Latin American Robotics Council He was the Chair for the Brazilian Committee on Robotics and on Computer Graphics and Image Processing, both under the Brazilian Computer Society. 